\title{
High-Throughput Fragment Docking into the BAZ2B Bromodomain: Efficient in Silico Screening for X-Ray Crystallography
}

\author{
Graziano Lolli* and Amedeo Caflisch* \\ Department of Biochemistry, University of Zürich, Winterthurerstrasse 190, CH-8057, Zürich, Switzerland \\ Supporting Information
}

ABSTRACT: Bromodomains are protein modules that bind to acetylated lysine side chains in histones and other proteins. The bromodomain adjacent to zinc finger domain protein $2 \mathrm{~B}$ (BAZ2B) has been reported to be poorly druggable. Here, we screened an in-house library of 350 fragments by automatic docking to the BAZ2B bromodomain. The top 12 fragments according to the predicted binding energy were selected for experiments of soaking into apo crystals of BAZ2B which yielded the structure of the complex for four of them, which is a hit rate of $33 \%$. Additional crystal structures were solved for BAZ2B and two scaffolds identified by analogy. For three topologically similar fragments, the crystal structures reveal binding modes with different penetration, i.e., with zero, one, and two water molecules, respectively, located between the fragment and the side chain of a conserved tyrosine (Tyr 1901) in the bottom of the acetyl lysine pocket of BAZ2B. Furthermore, a remarkable stereoselectivity of the acetyl lysine pocket emerges from the crystal structures of the bromodomains of BAZ2B and SMARCA4 in complex with the chiral diol MPD (2-methyl-2,4-pentanediol).

$\mathrm{T}$ he human genome encodes for 46 bromodomaincontaining proteins, many of them of potential pharmacological interest for their involvement in cancer, inflammation, and neurological diseases. ${ }^{1,2}$ Bromodomains are epigenetic reader domains that recognize acetylated lysine (Kac) in histones and other proteins. They are currently being investigated for the development of Kac-competitive ligands. Potent inhibitors have been disclosed for the BET (bromo and extra terminal) family of bromodomains, which was proven to be druggable. ${ }^{3,4}$ Other bromodomains were instead classified as "difficult to drug." Among these, the BAZ2B bromodomain has an unusually small Kac-binding pocket and is considered one of the least druggable bromodomains. ${ }^{6}$ Examples of potent BAZ2B ligands have only been reported recently.,

In vitro fragment-based screening was previously performed on the BAZ2B bromodomain using the AlphaScreen approach, which resulted in a hit rate of $0.8 \%$. ${ }^{6}$ When the same fragment library was screened against BRD2-BD1 and CREBBP bromodomains, with better druggability profiles, hit rates were much higher, $1.8 \%$ and $6.1 \%$, respectively. ${ }^{6}$ For the ATAD2 bromodomain, another target predicted to be difficult to inhibit by small molecules, fragment screening performed by Harber et al. ${ }^{9}$ resulted in a hit rate of $0.1 \%$.

Here, we decided to take advantage of a very fast fragmentdocking program (called SEED and developed in the group of one of us $)^{10,11}$ to screen an in-house library of 350 small molecules. The main objective at the outset of this study was the efficient in silico selection of a subset of about a dozen fragments for crystallographic (soaking) experiments. Crucially, the SEED program for docking uses a force field for scoring and

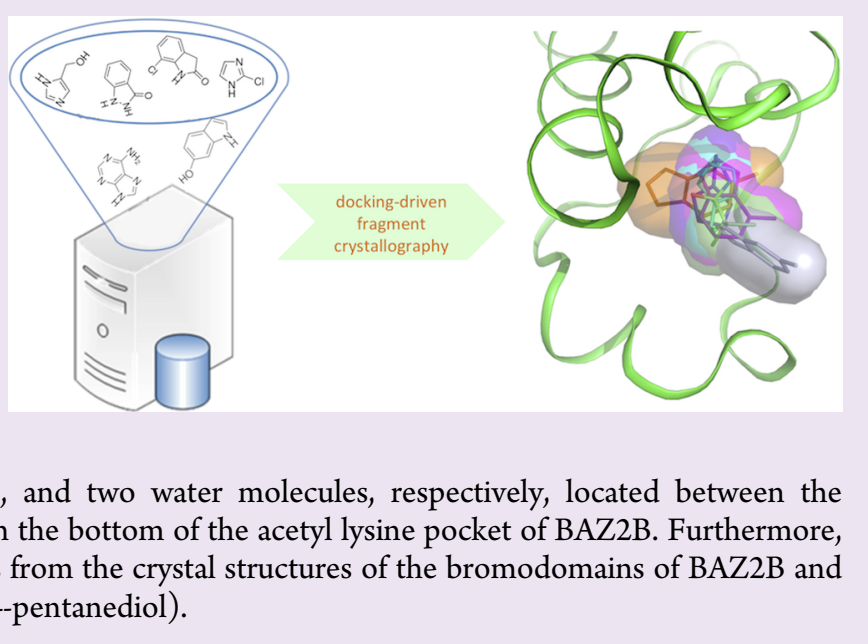

takes into account solvation effects. More precisely, the electrostatic desolvation penalty upon binding of the fragment to the protein is calculated for each pose and used for the final ranking. It is important to note that, despite the evaluation of a force-field energy with electrostatic solvation, SEED requires less than five seconds on a commodity processor for the docking and scoring of each fragment. The crystal structure in the complex with the BAZ2B bromodomain was solved for four of the 12 fragments prioritized by the SEED program (adenine, 2-chloro-imidazole, 4(5)-(hydroxymethyl)imidazole, and 6hydroxyindole). The hit rate of $33 \%$ provides evidence that fragment screening in silico is time- and resource-efficient.

We also report two additional BAZ2B structures, one in complex with a small molecule that mimics the Kac side chain ( $N$-methyltrimethylacetamide), the other with 6-aminoindazole, a compound structurally similar to 6-hydroxyindole but showing a more exposed binding mode which is different from the other tested fragments. The overall result is the identification of four different scaffolds (imidazole, purine, indole, and indazole) and three binding modes with different depths and numbers of conserved water molecules in the Kac binding site.

Finally, we present structures of MPD (2-methyl-2,4pentanediol) bound to BAZ2B and SMARCA4 bromodomains

Special Issue: Epigenetics

Received: November 6, 2015

Accepted: March 4, 2016

Published: March 4, 2016 
which show enantiomeric selectivity in different regions of their Kac binding pockets.

\section{RESULTS AND DISCUSSION}

Fragment Screening. All of the 350 fragments of an inhouse library were screened in silico. The initial library is fully unbiased as we did not make use of prior X-ray structural knowledge to enrich the screening library with groups likely to be active, e.g. the acetyl moiety present in many fragments found in the Kac binding site of bromodomains. ${ }^{6,12-14}$ The library, which was used previously for other projects, was built according to Hartshorn et al. ${ }^{15}$ and assembled with commercially available compounds.

The top 12 fragments according to the binding energy calculated by SEED were tested experimentally by soaking them in apo crystals of the BAZ2B bromodomain. Four of the 12 fragments were clearly identifiable in the Kac binding pocket (Figure 1 and Supporting Information Figure S1). Statistics on $\mathrm{X}$-ray diffraction data and final models are reported in Table 1.
F39<smiles>OCc1cnc[nH]1</smiles>

F59<smiles>Nc1ncnc(N)c1N</smiles>

F275<smiles>Clc1ncc[nH]1</smiles>

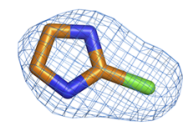

F60

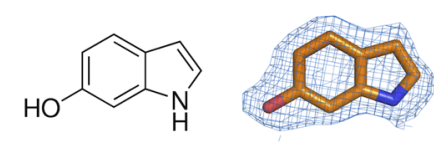

Figure 1. Fragments identified by docking into the Kac binding site of the BAZ2B bromodomain and validated by X-ray crystallography. $2 F_{\mathrm{o}}-F_{\mathrm{c}}$ map is contoured at $1 \sigma$.

Fragment F39 (4(5)-(hydroxymethyl)imidazole) forms a $\mathrm{H}$ bond between its N1 nitrogen atom and the carbonyl oxygen of Pro1888 (Figure 2a). Furthermore, its N3 nitrogen acts as a $\mathrm{H}$ bond acceptor for the side chain $\mathrm{NH}_{2}$ of Asn1944 and the conserved water molecule $\mathrm{W} 1$ (water molecules are named accordingly to ref 16) which in turn acts as a H-bond acceptor for the hydroxyl group of Tyr1901. The H bond with W1 is shorter than the one with the Asn1944 side chain (2.8 and 3.1 $\AA$, respectively), suggesting that the former is stronger. An additional $\mathrm{H}$ bond between the F39 hydroxyl group and the side chain oxygen of Asn1944 is present. F39 binds to BAZ2B similarly to 2-(hydroxymethyl)-6-methylpyridin-3-ol (PDB 4CUR) whose binding mode has not been described in any publication (Figure 2b). Importantly, the pose and hydrogen bonds of the imidazole ring of F39 predicted by docking are in agreement with the crystal structure. On the other hand, the hydroxymethyl substituent points into solvent instead of donating the $\mathrm{H}$ bond to the Asn 1944 side chain (Figure 2a). This discrepancy is due to the different tautomerization states used for docking and observed in the crystal structure which have the hydroxymethyl substituent in positions 5 and 4, respectively. Thus, the nitrogen atom ortho to the substituent was considered as a hydrogen bond donor during docking, whereas it is a hydrogen bond acceptor in the crystal structure. Similarly, the nitrogen atom that was meta was considered as a hydrogen bond acceptor during docking, whereas it is a hydrogen bond donor in the X-ray structure.

A second F39 molecule binds on the BAZ2B surface far away from the Kac pocket (Supporting Information Figure S2). It stacks on the His 1883 side chain and H-bonds to the Glu1884 main chain nitrogen via its N3. Its hydroxyl group H-bonds to the Tyr1960 side chain and to a water molecule whose tetrahedral coordination is filled by Thr1882 and Tyr1960 side chains and by Glu1879 main chain. Its lower occupancy and higher B-factors are indicative of poor binding affinity.

Fragment F59 (adenine, 6-aminopurine) binds into the BAZ2B Kac pocket posing its imidazole ring similarly to F39 with the $\mathrm{H}$ bond to Pro1888 (Figure 2c). A slightly different orientation of $\mathrm{F} 59$ however renders the W1-mediated $\mathrm{H}$ bond to Tyr1901 stronger with respect to F39, while the geometry of the direct $\mathrm{H}$ bond to Asn 1944 is less favorable than in the structure with F39. Indeed, the N3 nitrogen of the aminepyrimidine ring is closer to Asn1944 nitrogen (and almost at $\mathrm{H}$ bond distance to it) than the $\mathrm{N} 9$ nitrogen of the imidazole ring. F59 adapts nicely to the surrounding water network with water W5 being coordinated by both N7 and the amine group. It is interesting to note that the pose predicted by docking is identical to the binding mode observed in the crystal structure (Figure 2c). During the writing of this manuscript, a few structures of fragments bound to BAZ2B have been deposited in the PDB, without any accompanying publication. Among these, 2-amino-6-chlorobenzothiazole (PDB 5CU8) binds similarly to F59, without H-bonding to Asn1944 (Figure 2d). Instead, the closely related 6-chloropurine (PDB 5CUD) is rotated by approximately $40^{\circ}$ and in $\mathrm{H}$-bond contact with Asn1944 through the imidazole nitrogen. 2-Aminopurine fragments were described also in complex with BRD4 and BRD9 (PDB 4XY8, 4XY9, and 4XYA). ${ }^{17}$ Compared to F59 (Figure $2 \mathrm{~d}$ ), these larger fragments bind slightly more deeply in the Kac pocket interacting with the conserved Asn via the amine-pyrimidine and not the imidazole ring.

The N3 nitrogen atom of fragment F275 (2-chloroimidazole) is involved in $\mathrm{H}$-bonds of similar length with the side chain $\mathrm{NH}_{2}$ of Asn1944 (direct) and hydroxyl group of Tyr1901 (W1-mediated; Figure 2e). Its N1 nitrogen forms a W5-mediated $\mathrm{H}$ bond to the Pro1888 carbonyl group. The chlorine substituent of fragment F275 points in a direction opposite to the hydroxymethyl substituent of F39 (Figure 2a and e). In this orientation, the chlorine atom of F275 nicely fits the position occupied by the Kac methyl group, having very similar sizes. To the best of our knowledge, a halogen has been used to fill this region only in another case, the first bromodomain of BRD4 in complex with a chloro-pyridone ligand (PDB $4 \mathrm{MEP}{ }^{18}$ Figure $2 \mathrm{f}$ ). It would be interesting to understand whether the presence in this position of chlorine or other halogens and their polarizing effect could be advantageous in the development of new bromodomain inhibitors. Again, docking was successful for this fragment (Figure 2e).

The interactions described for F275 closely resemble those observed for $\mathrm{N}$-methyltrimethylacetamide (NMTA) bound to the first bromodomain of BRD4 (PDB 4IOO). ${ }^{19}$ Prompted by this observation, we also determined the structure of this small 
Table 1. Data Collection and Refinement Statistics

\begin{tabular}{|c|c|c|c|c|c|c|c|c|}
\hline & BAZ2B/F39 & BAZ2B/F59 & BAZ2B/F60 & BAZ2B/F275 & BAZ2B/F103 & BAZ2B/NMTA & BAZ2B/MPD & $\begin{array}{c}\text { SMARCA4/ } \\
\text { MPD }\end{array}$ \\
\hline \multicolumn{9}{|c|}{ data collection } \\
\hline space group & $C 222_{1}$ & $C 222_{1}$ & $C 222_{1}$ & $\mathrm{C} 222_{1}$ & $C 222_{1}$ & $C 222_{1}$ & $\mathrm{C} 222_{1}$ & $\mathrm{P}_{5}$ \\
\hline \multirow{3}{*}{$\begin{array}{l}\text { unit-cell } \\
\text { parameters }(\AA)\end{array}$} & $a=80.79$ & $a=81.13$ & $a=83.03$ & $a=81.33$ & $a=80.92$ & $a=81.37$ & $a=81.32$ & $a=86.78$ \\
\hline & $b=96.15$ & $b=96.24$ & $b=96.04$ & $b=96.18$ & $b=96.21$ & $b=96.11$ & $b=96.19$ & $b=86.78$ \\
\hline & $c=57.77$ & $c=57.78$ & $c=57.90$ & $c=57.79$ & $c=57.78$ & $c=57.81$ & $c=57.75$ & $c=97.96$ \\
\hline $\mathrm{X}$-ray source & PXI, SLS & PXI, SLS & XRD1, Elettra & PXIII, SLS & PXIII, SLS & PXIII, SLS & PXI, SLS & PXIII, SLS \\
\hline wavelength $(\AA)$ & 1.000 & 1.000 & 0.915 & 0.979 & 0.979 & 1.000 & 1.000 & 1.000 \\
\hline \multirow[t]{2}{*}{ resolution ( } & $48.07-1.65$ & $48.12-1.85$ & $48.02-1.96$ & $48.09-2.07$ & $48.10-1.90$ & $48.06-1.78$ & $48.10-1.65$ & $43.39-2.00$ \\
\hline & $(1.68-1.65)$ & $(1.89-1.85)$ & $(2.01-1.96)$ & $(2.18-2.07)$ & $(1.94-1.90)$ & $(1.87-1.78)$ & $(1.68-1.65)$ & $(2.05-2.00)$ \\
\hline$R_{\text {merge }}(\%)$ & $5.4(62.1)$ & $8.3(73.3)$ & $3.7(34.8)$ & $4.8(39.6)$ & $5.1(71.5)$ & $3.1(45.5)$ & $4.3(63.3)$ & $8.3(67.8)$ \\
\hline$R_{\text {meas }}(\%)$ & $5.9(67.2)$ & $9.0(79.8)$ & $4.0(37.6)$ & $5.4(44.8)$ & $5.6(77.6)$ & $3.8(54.0)$ & $4.6(68.6)$ & $9.8(79.3)$ \\
\hline$R_{\text {pim }}(\%)$ & $2.3(25.5)$ & $3.5(31.2)$ & $1.6(14.1)$ & $1.9(15.1)$ & $2.2(29.7)$ & $1.5(21.0)$ & $1.8(26.3)$ & $5.0(40.7)$ \\
\hline$<I / \sigma(I)>$ & $15.4(2.4)$ & $11.8(2.2)$ & $32.0(4.4)$ & $26.3(6.1)$ & $18.2(2.4)$ & $28.8(4.0)$ & $20.2(2.9)$ & $13.1(2.1)$ \\
\hline completeness (\%) & $99.9(100)$ & $99.8(99.6)$ & $97.2(99.6)$ & $99.6(97.8)$ & $99.7(99.3)$ & $99.7(99.0)$ & $99.9(99.9)$ & $99.6(98.0)$ \\
\hline \multirow[t]{2}{*}{ multiplicity } & $6.5(6.6)$ & $6.4(6.3)$ & $6.5(6.9)$ & $8.6(8.5)$ & $6.4(6.7)$ & $6.4(6.5)$ & $6.6(6.7)$ & $3.6(3.7)$ \\
\hline & & & & refinement & & & & \\
\hline resolution $(\AA)$ & $48.07-1.65$ & $42.29-1.85$ & $41.52-1.96$ & $48.09-2.07$ & $48.10-1.90$ & $48.06-1.78$ & $42.30-1.65$ & $43.39-2.00$ \\
\hline \multirow[t]{2}{*}{$R_{\text {work }} / R_{\text {free }}(\%)$} & $17.6 / 19.7$ & $17.6 / 20.2$ & $18.8 / 21.7$ & $19.4 / 21.9$ & $17.9 / 21.2$ & $17.4 / 19.8$ & $17.8 / 20.4$ & $19.2 / 22.8$ \\
\hline & & & & RMS deviations & & & & \\
\hline bond lengths $(\AA)$ & 0.006 & 0.007 & 0.007 & 0.007 & 0.007 & 0.006 & 0.006 & 0.004 \\
\hline bond angles (deg) & 1.1 & 1.0 & 1.1 & 1.1 & 1.0 & 1.1 & 1.0 & 0.9 \\
\hline PDB entry & $5 \mathrm{DYU}$ & SDYX & 5E9I & 5E9K & 5E9L & $5 \mathrm{E} 9 \mathrm{M}$ & 5E9Y & 5EA1 \\
\hline
\end{tabular}

Kac mimicking molecule in complex with BAZ2B and found it binds less deeply than in BRD4 (Supporting Information Figure S3). This is mainly due to the difference in sequence and structure between the two proteins at the level of the ZA loop and especially by the difference in side chain length between Val1898 in BAZ2B and Leu94, which is the corresponding residue in BRD4. These residues are in hydrophobic contact with the trimethyl moiety, and the more bulky leucine pushes NMTA closer to W1 than to Asn1944 in BRD4, rendering the W1-mediated H-bond to Tyr1901 the stronger interaction. In BAZ2B, the two interactions are more similar as observed for F275 (Supporting Information Figure S3). Different fragments containing the $\mathrm{N}$-methylacetamide moiety in complex with BAZ2B have been deposited recently in the PDB (5CUE, 5CQ7, 5CQA); in the first two, $N$-methylacetamide superposes almost perfectly to NMTA, while in the last it moves more deeply inside the cavity as observed in BRD4, again for steric constraints (Supporting Information Figure S3).

Fragment F60 (6-hydroxyindole) shows a peculiar binding mode to the BAZ2B Kac pocket. The F60 hydroxyl group Hbonds to the Pro1888 main chain oxygen. However, it does not interact with Asn1944 nor with the conserved W1. Instead W1 is displaced by F60 and its position roughly occupied by the indole nitrogen that forms a W2-mediated $\mathrm{H}$ bond to the main chain carbonyl of Val1936 (Figure 3a). The side chain of Tyr1901 is shifted by F60 with respect to its orientation in the three complexes discussed above (about $2.4 \AA$ for the hydroxyl oxygen) so that its side chain now $\mathrm{H}$-bonds with a different water molecule. F60 binds deeper into the cavity with respect to the other fragments: its buried surface is $244 \AA^{2}$ over a total surface of $272 \AA^{2}$ (90\% compared to $209 / 264 \AA^{2}=79 \%$ for F59). As a consequence, F60 is in van der Waals contact with Val1936, Asn1939, and Cys1940 in addition to all others amino acids contacted by F59. This is the first example of a compound able to displace a water molecule in BAZ2B, although the displacement of structural water molecules in the Kac-binding site have been predicted by MD simulations of spontaneous
Kac binding ${ }^{20}$ and later reported for compounds binding to $\mathrm{BRD} 4, \mathrm{ATAD} 2$, and $\mathrm{PB} 1 .^{21-24}$ This structure, together with the one presented below of the SMARCA4 bromodomain in complex with MPD, reinforces the idea that similar water displacement might be possible in many bromodomains. Indeed, during the writing of this manuscript, structures of the PFI-3 chemical probe in complex with SMARCA2 and SMARCA4 have been released (PDB 5DKC and 5DKD). As in the case of PB1, this compound is able to displace the conserved water molecules from the deepest region of the Kac binding pocket. Moreover, a deeper search in the PDB database returned the structure of BRD1 in complex with a tetrahydroindazole derivative (PDB 5AMF). The binding pose of this compound in $\mathrm{BRD} 1$, also displacing only the $\mathrm{W} 1$ water molecule, is similar to the observed binding of F60 in BAZ2B (Figure 3b). The docking pose of F60 is less buried than the one observed in the crystal structure. This discrepancy is due to the W1 water molecule in the BAZ2B structure that was used for docking because the docking procedure treats the protein structure as rigid and preserves the position and orientation of the structural waters.

Intrigued by this discrepancy, we decided to test F103 which is the compound most similar to F60 in our library. Fragment F103 (6-aminoindazole) binds in the Kac pocket of BAZ2B but with a binding mode surprisingly different from both F60 and the other tested fragments. In particular, while F60 binds more deeply compared to the "usual" binding mode (the one of Kac and all BAZ2B ligands reported as of today), F103 shows an opposite preference for a significantly more external pose (Figure 3c and d). The N1 atom of F103 acts as donor for the oxygen atom of the Asn 1944 side chain. On the other hand, F103 does not form direct hydrogen bonds with W1 nor with the side chain nitrogen of Asn1944. Hydrogen bonds are instead mediated by a water molecule (W8) that is tetrahedrally coordinated by F103 N2 nitrogen, Asn 1944 side chain nitrogen, and the two conserved water molecules W1 and W5 (in turn in hydrogen bond contacts with Tyr1901 and 

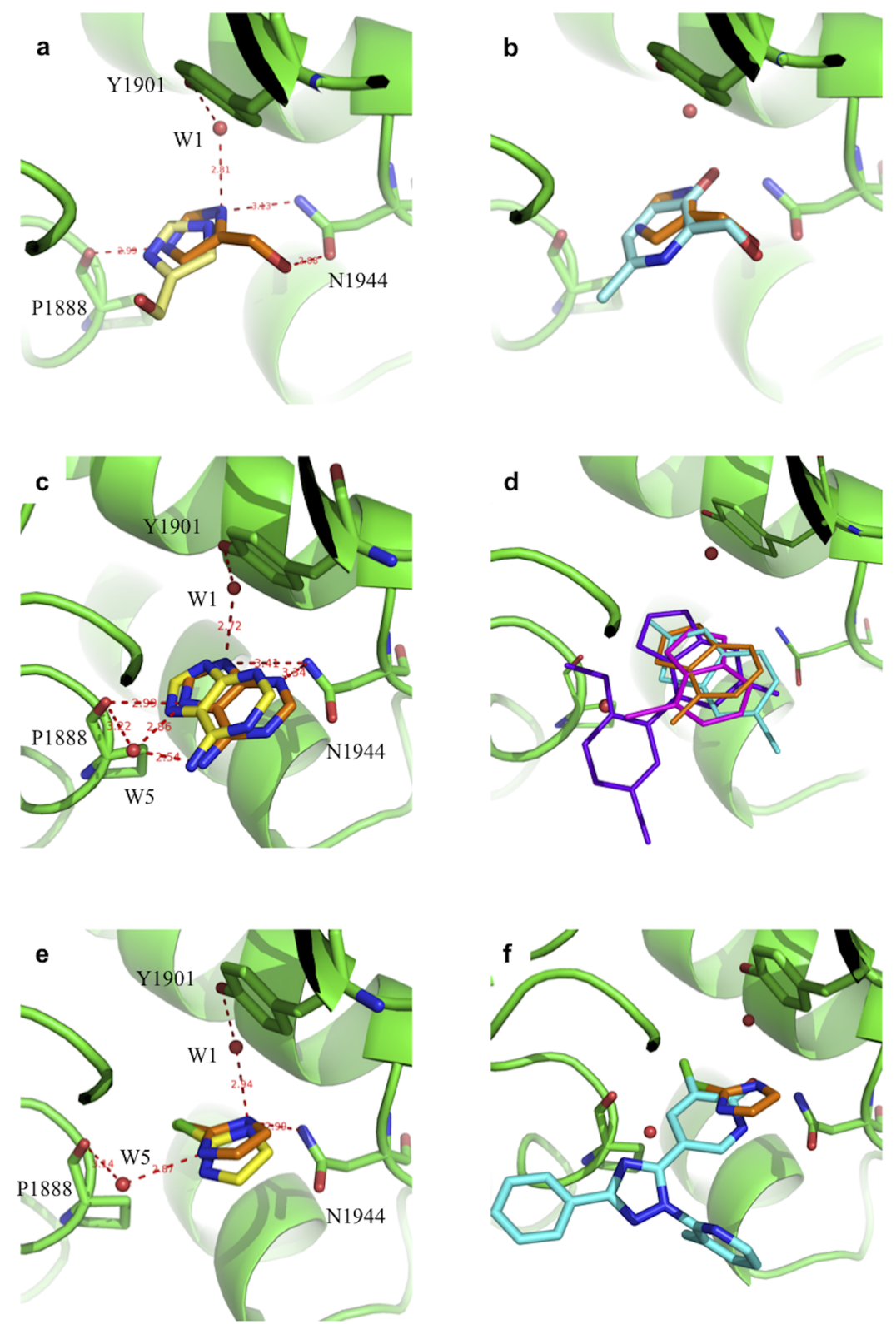

Figure 2. Binding mode of fragments F39, F59, and F275 to the BAZ2B Kac-pocket. (a) F39 in the crystal structure (carbon atoms in orange) is superposed to its docking pose (yellow). Intermolecular hydrogen bonds are shown for the crystal structure (dashed lines). (b) Comparison between binding modes of F39 and 2-(hydroxymethyl)-6-methylpyridin-3-ol (cyan, PDB 4CUR). (c) F59 binds to BAZ2B very similarly to what was predicted by in silico screening. Color code same as in a. (d) Binding mode of F59 (orange) is more similar to that of 2-amino-6-chlorobenzothiazole (cyan, PDB 5CU8) than to the one exhibited by 6-chloropurine (magenta, PDB 5CUD). Binding of a 2-amine-purine derivative to BRD4 is shown in purple (PDB 4XY9). (e) Experimental and predicted binding modes for F275 are essentially identical. Color code same as in a. (f) Chlorine fits in the position occupied by the Kac methyl group both in the BAZ2B/F275 complex and in the complex of BRD4 with a chloro-pyridone derivative (cyan, PDB 4MEP).

Pro1888, respectively). It is interesting to note that indazole (F18, Table S1) does not seem to bind which might be due to its slightly lower solubility with respect to 6-aminoindazole and/or to the increased polarity of the solvent exposed aniline ring.

Overall, fragment screening by docking followed by X-ray crystallography has resulted in the identification of BAZ2B ligands with four different scaffolds (imidazole, purine, indole, and indazole). These scaffolds consist of 6 to 10 heavy atoms and are thus expected to bind in the millimolar to highmicromolar range. They are interesting starting points for hitfragment optimization by growing. Importantly, the binding mode predicted by docking is correct for three of the four fragments (adenine, 2-chloro-imidazole, and 4(5)(hydroxymethyl)imidazole). On the other hand, the predicted binding mode of 6-hydroxyindole is different with respect to the crystal structure because this fragment displaces a structural water molecule which is present in the vast majority of crystal structures of bromodomain/ligand complexes. The five crystal structures reveal three different levels of fragment burial, which can be referred to as 0 -water, 1 -water, and 2 -water binding modes, respectively. These three abbreviations are used in the following to indicate the number of water molecules located between the fragment and the hydroxyl group of the conserved 

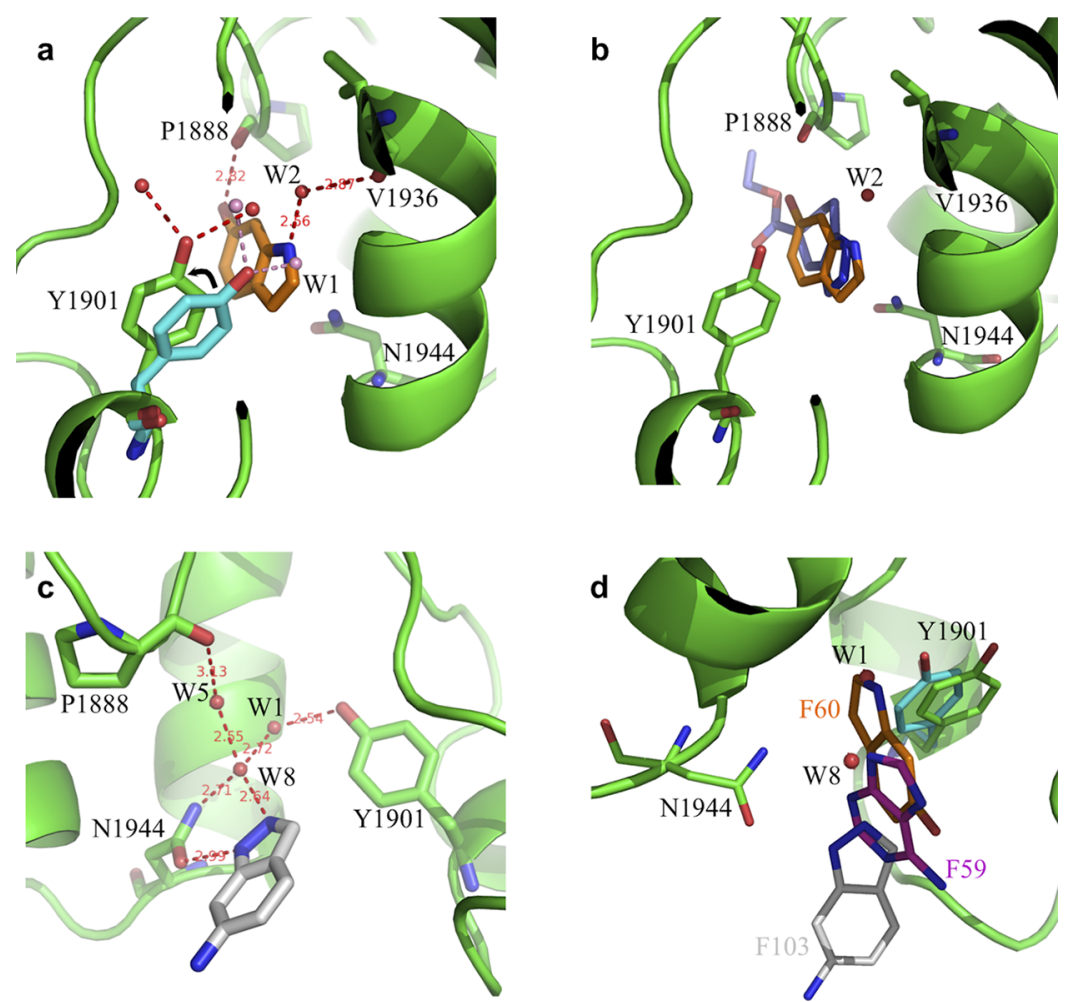

Figure 3. Unusual binding modes of fragments F60 and F103 to the BAZ2B bromodomain. (a) The binding of F60 displaces the W1 water molecule. In the crystal structure of the complex between BAZ2B (green, water molecules in red) and F60 (orange), the side chain of Tyr1901 moves away from its usual position in hydrogen bond contact with W1 forming new interactions (red dashed lines) with the surrounding solvent. As a basis of comparison, the orientation of the side chain of Y1901 (cyan) and position of water molecules (pink) of the crystal structure of apo BAZ2B are also shown. They originate from a structural overlap with the F60/BAZ2B structure. (b) The binding mode of F60 (orange) to BAZ2B (green) is similar to that of ethyl 4,5,6,7-tetrahydro-1H-indazole-5-carboxylate (light blue) to BRD1. (c) F103 (gray) is involved as a donor in a hydrogen bond with the side chain carbonyl of Asn194. It is also connected to the carbonyl of Pro1888 and hydroxyl of Tyr1901 through a well-ordered water network. (d) Different binding modes of fragments in the BAZ2B Kac pocket: the deep, 0-water binding mode is exploited by F60 (orange); F59 (magenta) adopts the "usual" 1-water binding mode; F103 (gray) binds more externally in the 2-water binding mode.
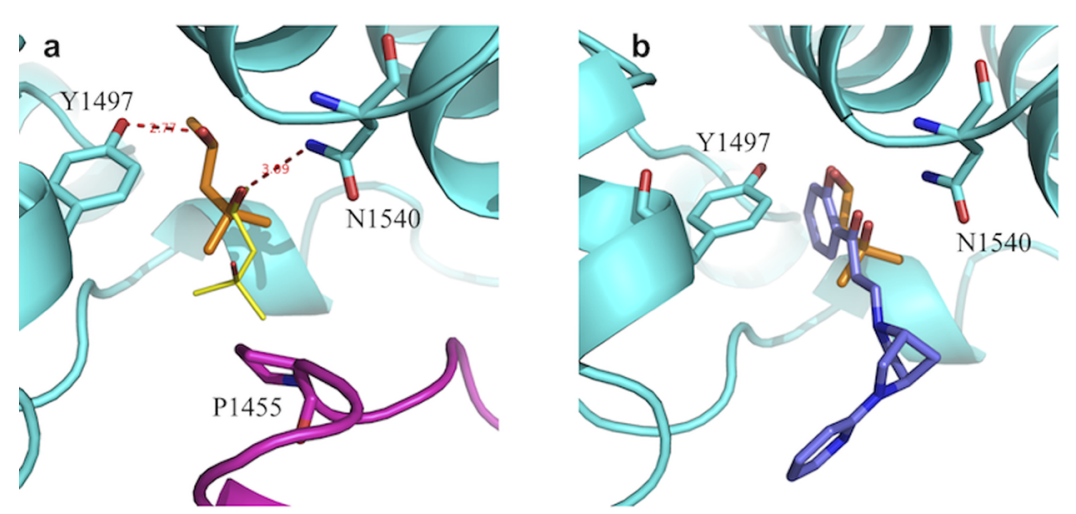

Figure 4. Two binding modes of MPD and stereoselectivty in the SMARCA4 bromodomain. (a) Pro1455 from a symmetric chain (magenta) pushes the (R)-(+) enantiomer of MPD (orange) in the 0-water binding mode. In this deep binding mode, MPD displaces the W1 water molecule and interacts directly with Tyr1497. In contrast, the (S)-(-) enantiomer of MPD is preferred in the 1-water binding mode (yellow), which is observed in two of the three protein chains in the asymmetric unit. (b) MPD in the 0-water binding mode shows the same polar interactions as in the SMARCA4/PFI-3 complex (PFI-3 in light blue).

Tyr1901 at the bottom of the Kac binding site: no water for the "deep" binding mode observed for F60 (Figure 3a), one water molecule which bridges the fragment and the Tyr1901 hydroxyl for the most frequent binding mode (Figure 2a, c, and e), and two water molecules as observed for F103 (Figure 3c).

Effect of Cosolvent and Stereoselectivity. Crystals of the BAZ2B bromodomain were grown in the presence of MPD, which was also found in its Kac binding pocket. A soaking protocol was then designed to avoid competition of MPD with fragments. Crystals were transferred in a soaking solution containing the tested fragment but devoid of MPD, so that soaking of the fragment is achieved at the same time with MPD back-soaking.

MPD forms hydrogen bonds of similar length between its C4-hydroxyl group and both W1 and the Asn1944 side chain nitrogen. The C2-hydroxyl is instead engaged in a hydrogen 
bond with Asn 1944 side chain oxygen (Supporting Information Figure S4a). MPD binding is stereospecific. The (R)-(+) enantiomer is completely excluded during the refinement procedure with $100 \%$ occupancy assigned to the (S)-(-) enantiomer. Specificity can be assigned to the better fitting of the $(S)-(-)$ enantiomer with the water network in the binding cavity, which would need to rearrange in order to comfortably accommodate the other enantiomer.

We also determined the structure of the SMARCA4 bromodomain with bound MPD. In the three protein chains in the asymmetric unit, both 0 -water and 1-water binding modes are present (Figure 4a). In chains A and C, MPD forms hydrogen bonds with both Asn 1540 side chain nitrogen and Tyr1497 side chain oxygen (W1 mediated; Supporting Information Figure S4b). In chain B, MPD displaces the water molecules from the bottom of the cavity, roughly occupying W1 positions with its C4-hydroxyl group; MPD forms direct $\mathrm{H}$ bonds to the Asn 1540 side chain nitrogen and Tyr1497 side chain oxygen (Figure 4a, MPD in orange). The 0water binding mode of MPD to chain B is stabilized by the crystal packing. Pro1455 from a symmetric chain faces the chain B Kac binding pocket pushing MPD deeply in the cavity (Figure 4a). Thus, the 0 -water binding mode of MPD is likely to be less populated in solution than the 1-water mode, as the latter is not influenced by crystal contacts. On the other hand, the 0-water binding reflects that observed for the PFI-3 chemical probe, with the two MPD hydroxyls superimposing to the hydroxyl and carbonyl oxygens of PFI-3 (Figure 4b).

Interestingly, the two binding modes of MPD in SMARCA4 show opposite stereoselectivity. The 0 -water binding mode is proper of the $(\mathrm{R})-(+)$ enantiomer. Refinement of the racemic mixture resulted in $94 \%$ occupancy for this enantiomer versus $6 \%$ for the (S)-(-) enantiomer (Supporting Information Figure $\mathrm{S} 4 \mathrm{c})$. This is mainly due to poorer interaction of the $(\mathrm{S})-(-)$ enantiomer with the side chain of Tyr1497 (H-bond distance increasing from 2.77 to $3.09 \AA$ while the asymmetric chiral carbon moves closer to the tyrosine hydroxyl group (from 3.55 to $3.12 \AA)$ ). In the 1 -water binding mode, the $(S)-(-)$ enantiomer is preferred. Occupancy is $65 \%$ for this enantiomer in chain A and $81 \%$ in chain C (MPD shows slightly different orientations in the two chains). In this case, poorer interaction is observed for the (R)-(+) enantiomer with the side chain of Asn 1540, with H-bond distance increasing from 3.03 to $3.26 \AA$ in chain $\mathrm{A}$ and from 2.88 to 3.46 in chain $\mathrm{C}$ (Supporting Information Figure $S 4 d$ ).

MPD was also found in the Kac binding pocket of the ATAD2 bromodomain where only the $(S)-(-)$ enantiomer is present (PDB 4QSR). This crystal structure shows the 1-water binding mode, but here MPD is rotated by $180^{\circ}$ with respect to the pose in BAZ2B and SMARCA4. As a consequence, MPD interacts with both Asn1944 and Tyr1901 (W1-mediated) via its C2-hydroxyl group, while the chiral C4 is now located more externally.

Stereoselectivity in the region facing the conserved asparagine has also been exploited in the potent inhibitors JQ1, I-BET762, and I-BET726 targeting a number of bromodomains, namely BRD2, BRD3, BRD4, and BRDT. With the structures of MPD bound bromodomains, the characterization of stereospecificity is extended to different families and to the region usually occupied by the deeply buried water molecule W1.

\section{CONCLUSIONS}

We have used fragment docking to identify scaffolds that bind to the BAZ2B bromodomain. The screening of a library of 350 fragments by docking with the in-house developed program SEED $^{10,11}$ took only $20 \mathrm{~min}$ on a single core of a commodity processor. The top 12 fragments according to the predicted binding energy were selected for soaking experiments into apo crystals of BAZ2B. The crystal structure of the complex with BAZ2B was solved for four of the 12 top fragments (adenine, 2chloro-imidazole, 4(5)-(hydroxymethyl)imidazole, and 6-hydroxyindole, Figures 2 and 3a). Additional structures were solved for BAZ2B and two fragments identified by analogy (6aminoindazole, Figure 3c; NMTA, Supporting Information Figure S3) as well as the chiral diol MPD for which we solved the complex in BAZ2B and SMARCA4 (Figure 4 and Supporting Information Figure S4).

Three main results emerge from this study. First, fragment screening by docking is a very efficient procedure to prioritize fragments for X-ray crystallography. In silico screening followed directly by soaking seems more effective than frequently used approaches for in vitro screening (e.g., by AlphaScreen and/or thermal shift assay), at least for bromodomains. Importantly, the fragment-based docking approach has shown high hit rates for different bromodomains, viz., BRD4(1), ${ }^{25}$ CREBBP, ${ }^{26}$ BAZ2B (this study), and BRPF1 (Zhu and Caflisch, submitted), as well as for two subfamilies of tyrosine kinases. ${ }^{27}$ The SEED program for fragment docking is an efficient computational tool to prioritize fragments according to the evaluation of a physics-based (force field) energy which takes into account electrostatic desolvation effects. A significant limitation of the SEED program for fragment docking is the lack of flexibility in the protein binding site and structural water molecules. This limitation has resulted in a predicted binding mode of type 1-water for 6-hydroxyindole while the crystal structure shows that this fragment is fully buried in the 0-water binding mode. We plan to address this limitation in a new version of SEED which will require a longer, but still extremely fast, execution time. Concerning the eight false positives predicted by SEED, there are several possible reasons including wrong protonation state (see Supporting Information Figure S1), inaccuracies inherent to the force field (e.g., fixed partial charges and neglect of polarization effects), and/or the rigidprotein approximation. Related to the latter, entropic penalties of the solute are not taken into account for ranking the docked fragments. It is important to note in this context that the binding modes of small molecules can be different in crystal structures solved at $100 \mathrm{~K}$ and RT. ${ }^{28}$

Second, there are three possible binding modes in the Kac binding site of BAZ2B with two, one, or zero water molecules acting as bridges for hydrogen bonds with the side chain hydroxyl group of the conserved Tyr1901 in the ZA-loop (Figure 3d). These three binding modes have been observed for three fragments that are different but share the same double-ring topology (6-aminoindazole, adenine, and 6hydroxyindole). They show that the degree of burial of this double-ring scaffold in the Kac binding site is linked to the number of structured water molecules that separate it from the side chain hydroxyl of Tyr1901 at the bottom of the pocket. Figure $3 \mathrm{~d}$ indicates that the most buried fragment (6hydroxyindole) reaches its deep binding mode by transiently stepping through metastable states with two and one water molecules as observed for 6-aminoindazole and adenine, 
respectively. In other words, it is likely that the process of binding to the 0 -water site requires the progressive expulsion of two structural water molecules.

Third, only the (S)-(-) enantiomer of the chiral diol MPD binds to the BAZ2B bromodomain with one water molecule bridging the C4-hydroxyl of MPD and the hydroxyl of Tyr1901 (Supporting Information Figure S4a). In contrast, the crystal structure of the SMARCA4 bromodomain with MPD shows that the (R)-(+) and (S)-(-) enantiomers prefer the binding mode with zero and one water molecule acting as bridges, respectively. Thus, very specific interactions between the ligand and the narrow pocket of the Kac binding site result in a "racemic mixture filter" which could be used for de novo design and optimization of known ligands.

\section{METHODS}

Fragment Docking. The library of 350 fragments was docked into BAZ2B by the program SEED, ${ }^{10,11}$ which has already been used for the in silico identification of potent and selective BRD4(1) and CREBBP ligands. ${ }^{26,29}$ Here, a structure of BAZ2B in the complex with a 7-phenoxyindolizine derivative (PDB code $4 \operatorname{IR}^{8}$ ) was used for docking. The binding site was defined by the conserved asparagine (Asn 1944) and the structurally conserved water molecules. The partial charges and van der Waals parameters of the protein were taken from the CHARMM36 all-atom force field, ${ }^{30,31}$ while the fragments were parametrized using the CHARMM general force field (CGenFF). ${ }^{32}$ Importantly, CGenFF is fully consistent with the CHARMM36 force field. SEED takes into account electrostatic desolvation effects in the continuum dielectric approximation by means of the generalized Born approach. ${ }^{33}$ The dielectric constant was set to 2.0 and 78.5 in the lowdielectric region (solute) and high-dielectric region (solvent), respectively. The docking of the 350 fragments with SEED required only $20 \mathrm{~min}$ of a single core of an i7-930 processor at $2.8 \mathrm{GHz}$. The program SEED can be downloaded for free from http://www. biochem-caflisch.uzh.ch/download/.

Protein Production. BAZ2B bromodomain (aa. 1858-1972) and SMARCA4 bromodomain (aa. 1451-1580) were expressed and purified as described previously. ${ }^{34}$ Briefly, after induction (IPTG 0.5 $\mathrm{mM}$ for BAZ2B and $0.1 \mathrm{mM}$ for SMARCA4) and overnight expression at $18{ }^{\circ} \mathrm{C}$, the $6 \times$ His-tagged proteins were purified by coupled DEAEIMAC using step elution with increasing concentrations of imidazole. Buffer was exchanged and the $6 \times$ His tag removed with TEV protease. The bromodomains were further purified by a second IMAC and a SEC using a Superdex 75 column. Proteins were concentrated to 15 $\mathrm{mg} \mathrm{mL} \mathrm{m}^{-1}$ and frozen in liquid nitrogen.

Crystallization and Soaking. BAZ2B and SMARCA4 bromodomains were crystallized by vapor diffusion in sitting drops at $4{ }^{\circ} \mathrm{C}$. For BAZ2B, crystallization buffer ( $\mathrm{pH} 7.5$ ) is composed by PEGs of different lengths PEG500MME (18-21\%), PEG1000 (0-7\%), PEG3350 (0-7\%), PEG20000 (9-11\%), and also contains MPD $(0-7 \%)$. Overnight soaking of fragments was performed by transferring the apo crystals in a soaking solution composed of $32 \%$ PEG500MME and 16\% PEG20000 in which fragments were previously dissolved. Fragments were tested at $50 \mathrm{mM}$ if soluble at this concentration; less soluble compounds were tested at saturating concentrations. Soaked crystals were frozen in liquid nitrogen. SMARCA4 was crystallized at pH 6.5 in $12.5 \%$ PEG1000, $12.5 \%$ PEG3350, and $12.5 \%$ MPD.

Data Collection and Structure Solution. Diffraction data were collected at the Swiss Light Source, Paul Scherrer Institute (Villigen, Switzerland), beamlines PXI (F39, F59 and BAZ2B/MPD) and PXIII (F275, F103, NMTA and SMARCA4/MPD) and at the Elettra Synchrotron Light Source (Trieste, Italy), XRD1 beamline (F60). Data were processed with $\mathrm{XDS}^{35}$ and Aimless; ${ }^{36}$ structures were solved by molecular replacement with Phaser ${ }^{37}$ using PDB 4IR5 as a search model. Initial models were refined alternating cycles of automatic refinement with Phenix ${ }^{38}$ and manual model building with COOT. $^{39}$

\section{ASSOCIATED CONTENT}

\section{Supporting Information}

The Supporting Information is available free of charge on the ACS Publications website at DOI: 10.1021/acschembio.5b00914.

Figures S1-S4 (PDF)

\section{Accession Codes}

Structures were deposited to the PDB with accession numbers 5DYU (BAZ2B/F39), 5DYX (BAZ2B/F59), 5E9I (BAZ2B/ F60), 5E9K (BAZ2B/F275), 5E9L (BAZ2B/F103), 5E9M (BAZ2B/NMTA), 5E9Y (BAZ2B/MPD), and 5EA1 (SMARCA4/MPD).

\section{AUTHOR INFORMATION}

\section{Corresponding Authors}

*E-mail: graziano.lolli@bioc.uzh.ch.

*E-mail: caflisch@bioc.uzh.ch.

\section{Funding}

This work was supported in part by a grant of the Swiss Cancer League (Krebsliga Schweiz).

\section{Notes}

The authors declare no competing financial interest.

\section{ACKNOWLEDGMENTS}

We thank T. Knehans and J.-R. Marchand for the parametrization of the fragments, D. Spiliotopoulos (University of Zurich) for interesting discussions, and R. Battistutta (University of Padua) for his support. We are grateful to A. Widmer for his continuous support with the program WITNOTP, which was used for the visual analysis of docked poses and the comparison with the crystal structures. We also thank the staff at PXI and PXIII beamlines, Swiss Light Source, Paul Scherrer Institute (Villigen, Switzerland) and at XDR1 beamline, ELETTRA Synchrotron Light Source (Trieste, Italy) for on-site assistance.

\section{REFERENCES}

(1) Filippakopoulos, P., and Knapp, S. (2014) Targeting bromodomains: epigenetic readers of lysine acetylation. Nat. Rev. Drug Discovery 13, 337-356.

(2) Prinjha, R. K., Witherington, J., and Lee, K. (2012) Place your BETs: the therapeutic potential of bromodomains. Trends Pharmacol. Sci. 33, 146-153.

(3) Filippakopoulos, P., Qi, J., Picaud, S., Shen, Y., Smith, W. B., Fedorov, O., Morse, E. M., Keates, T., Hickman, T. T., Felletar, I., Philpott, M., Munro, S., McKeown, M. R., Wang, Y., Christie, A. L., West, N., Cameron, M. J., Schwartz, B., Heightman, T. D., La Thangue, N., French, C. A., Wiest, O., Kung, A. L., Knapp, S., and Bradner, J. E. (2010) Selective inhibition of BET bromodomains. Nature 468, 1067-1073.

(4) Nicodeme, E., Jeffrey, K. L., Schaefer, U., Beinke, S., Dewell, S., Chung, C.-w., Chandwani, R., Marazzi, I., Wilson, P., Coste, H., White, J., Kirilovsky, J., Rice, C. M., Lora, J. M., Prinjha, R. K., Lee, K., and Tarakhovsky, A. (2010) Suppression of inflammation by a synthetic histone mimic. Nature 468, 1119-1123.

(5) Vidler, L. R., Brown, N., Knapp, S., and Hoelder, S. (2012) Druggability analysis and structural classification of bromodomain acetyl-lysine binding sites. J. Med. Chem. 55, 7346-7359.

(6) Ferguson, F. M., Fedorov, O., Chaikuad, A., Philpott, M., Muniz, J. R., Felletar, I., von Delft, F., Heightman, T., Knapp, S., Abell, C., and Ciulli, A. (2013) Targeting low-druggability bromodomains: fragment based screening and inhibitor design against the BAZ2B bromodomain. J. Med. Chem. 56, 10183-10187. 
(7) Drouin, L., McGrath, S., Vidler, L. R., Chaikuad, A., Monteiro, O., Tallant, C., Philpott, M., Rogers, C., Fedorov, O., Liu, M., Akhtar, W., Hayes, A., Raynaud, F., Müller, S., Knapp, S., and Hoelder, S. J. (2015) Structure enabled design of BAZ2-ICR, a chemical probe targeting the bromodomains of BAZ2A and BAZ2B. J. Med. Chem. 58, 2553-2559.

(8) Chen, P., Chaikuad, A., Bamborough, P., Bantscheff, M., Bountra, C., Chung, C. W., Fedorov, O., Grandi, P., Jung, D., Lesniak, R., Lindon, M., Müller, S., Philpott, M., Prinjha, R., Rogers, C., Selenski, C., Tallant, C., Werner, T., Willson, T. M., Knapp, S., and Drewry, D. H. (2015) Discovery and Characterization of GSK2801, a Selective Chemical Probe for the Bromodomains BAZ2A and BAZ2B. J. Med. Chem. 59, 1410.

(9) Harner, M. J., Chauder, B. A., Phan, J., and Fesik, S. W. (2014) Fragment-based screening of the bromodomain of ATAD2. J. Med. Chem. 57, 9687-9692.

(10) Majeux, N., Scarsi, M., Apostolakis, J., Ehrhardt, C., and Caflisch, A. (1999) Exhaustive docking of molecular fragments with electrostatic solvation. Proteins: Struct., Funct., Genet. 37, 88-105.

(11) Majeux, N., Scarsi, M., and Caflisch, A. (2001) Efficient electrostatic solvation model for protein-fragment docking. Proteins: Struct., Funct., Genet. 42, 256-268.

(12) Chung, C.-W., Dean, A. W., Woolven, J. M., and Bamborough, P. (2012) Fragment-based discovery of bromodomain inhibitors part 1: inhibitor binding modes and implications for lead discovery. J. Med. Chem. 55, 576-586.

(13) Sachchidanand, Resnick-Silverman, L., Yan, S., Mutjaba, S., Liu, W. J., Zeng, L., Manfredi, J. J., and Zhou, M. M. (2006) Target structure- based discovery of small molecules that block human p53 and CREB binding protein association. Chem. Biol. 13, 81-90.

(14) Hay, D. A., Rogers, C., Fedorov, O., Tallant, C., Martin, S., Monteiro, O., Muller, S., Knapp, S., Schofield, C. J., and Brennan, P. E. (2015) Design and synthesis of potent and selective inhibitors of BRD7 and BRD9 bromodomains. MedChemComm 6, 1381-1386.

(15) Hartshorn, M. J., Murray, C. W., Cleasby, A., Frederickson, M., Tickle, I. J., and Jhoti, H. (2005) Fragment-based lead discovery using X-ray crystallography. J. Med. Chem. 48, 403-413.

(16) Huang, D., Rossini, E., Steiner, S., and Caflisch, A. (2014) Structured water molecules in the binding site of bromodomains can be displaced by cosolvent. ChemMedChem 9, 573-579.

(17) Picaud, S., Strocchia, M., Terracciano, S., Lauro, G., Mendez, J., Daniels, D. L., Riccio, R., Bifulco, G., Bruno, I., and Filippakopoulos, P. (2015) 9H-purine scaffold reveals induced-fit pocket plasticity of the BRD9 bromodomain. J. Med. Chem. 58, 2718-2736.

(18) Vidler, L. R., Filippakopoulos, P., Fedorov, O., Picaud, S., Martin, S., Tomsett, M., Woodward, H., Brown, N., Knapp, S., and Hoelder, S. (2013) Discovery of novel small-molecule inhibitors of BRD4 using structure-based virtual screening. J. Med. Chem. 56, 80738088.

(19) Lolli, G., and Battistutta, R. (2013) Different orientations of low-molecular-weight fragments in the binding pocket of a BRD4 bromodomain. Acta Crystallogr., Sect. D: Biol. Crystallogr. 69, 21612164.

(20) Magno, A., Steiner, S., and Caflisch, A. (2013) Mechanism and Kinetics of Acetyl-Lysine Binding to Bromodomains. J. Chem. Theory Comput. 9, 4225-4232.

(21) Harner, M. J., Chauder, B. A., Phan, J., and Fesik, S. W. (2014) Fragment-Based Screening of the Bromodomain of ATAD2. J. Med. Chem. 57, 9687-9692.

(22) Ember, S. W., Zhu, J. Y., Olesen, S. H., Martin, M. P., Becker, A., Berndt, N., Georg, G. I., and Schonbrunn, E. (2014) Acetyl-lysine binding site of bromodomain-containing protein 4 (BRD4) interacts with diverse kinase inhibitors. ACS Chem. Biol. 9, 1160-1171.

(23) Zhao, L., Cao, D., Chen, T., Wang, Y., Miao, Z., Xu, Y., Chen, W., Wang, X., Li, Y., Du, Z., Xiong, B., Li, J., Xu, C., Zhang, N., He, J., and Shen, J. (2013) Fragment-based drug discovery of 2thiazolidinones as inhibitors of the histone reader BRD4 bromodomain. J. Med. Chem. 56, 3833-3851.

(24) Fedorov, O., Castex, J., Tallant, C., Owen, D. R., Martin, S., Aldeghi, M., Monteiro, O., Filippakopoulos, P., Picaud, S., Trzupek, J.
D., Gerstenberger, B. S., Bountra, C., Willmann, D., Wells, C., Philpott, M., Rogers, C., Biggin, P. C., Brennan, P. E., Bunnage, M. E., Schüle, R., Günther, T., Knapp, S., and Müller, S. (2015) Selective targeting of the BRG/PB1 bromodomains impairs embryonic and trophoblast stem cell maintenance. Sci. Adv. 1, e1500723.

(25) Zhao, H., Gartenmann, L., Dong, J., Spiliotopoulos, D., and Caflisch, A. (2014) Discovery of BRD4 bromodomain inhibitors by fragment-based high-throughput docking. Bioorg. Med. Chem. Lett. 24, 2493-2496.

(26) Xu, M., Unzue, A., Dong, J., Spiliotopoulos, D., Nevado, C., and Caflisch, A. (2016) Discovery of CREBBP Bromodomain Inhibitors by High-Throughput Docking and Hit Optimization Guided by Molecular Dynamics. J. Med. Chem. 59, 1340.

(27) Zhao, H., and Caflisch, A. (2015) Molecular dynamics in drug design. Eur. J. Med. Chem. 91, 4-14.

(28) Fischer, M., Shoichet, B. K., and Fraser, J. S. (2015) One Crystal, Two Temperatures: Cryocooling Penalties Alter Ligand Binding to Transient Protein Sites. ChemBioChem 16, 1560-1564.

(29) Unzue, A., Xu, M., Dong, J., Wiedmer, L., Spiliotopoulos, D. Caflisch, A., and Nevado, C. (2016) Fragment-Based Design of Selective Nanomolar Ligands of the CREBBP Bromodomain. J. Med. Chem. 59, 1350.

(30) MacKerell, A. D., Bashford, D., Bellott, M., Dunbrack, R. L., Evanseck, J. D., Field, M. J., Fischer, S., Gao, J., Guo, H., Ha, S., Joseph-McCarthy, D., Kuchnir, L., Kuczera, K., Lau, F. T., Mattos, C., Michnick, S., Ngo, T., Nguyen, D. T., Prodhom, B., Reiher, W. E., Roux, B., Schlenkrich, M., Smith, J. C., Stote, R., Straub, J., Watanabe, M., Wiórkiewicz-Kuczera, J., Yin, D., and Karplus, M. (1998) All-atom empirical potential for molecular modeling and dynamics studies of proteins. J. Phys. Chem. B 102, 3586-3616.

(31) MacKerell, A. D., Jr, Feig, M., and Brooks, C. L., 3rd (2004) Improved treatment of the protein backbone in empirical force fields. J. Am. Chem. Soc. 126, 698-699.

(32) Vanommeslaeghe, K., Hatcher, E., Acharya, C., Kundu, S., Zhong, S., Shim, J., Darian, E., Guvench, O., Lopes, P., Vorobyov, I., and Mackerell, A. D., Jr. (2010) CHARMM general force field: A force field for drug-like molecules compatible with the CHARMM all-atom additive biological force fields. J. Comput. Chem. 31, 671-690.

(33) Scarsi, M., Apostolakis, J., and Caflisch, A. (1997) Continuum Electrostatic Energies of Macromolecules in Aqueous Solutions. J. Phys. Chem. A 101, 8098-8106.

(34) Filippakopoulos, P., Picaud, S., Mangos, M., Keates, T., Lambert, J. P., Barsyte-Lovejoy, D., Felletar, I., Volkmer, R., Muller, S., Pawson, T., Gingras, A. C., Arrowsmith, C. H., and Knapp, S. (2012) Histone recognition and large-scale structural analysis of the human bromodomain family. Cell 149, 214-231.

(35) Kabsch, W. (2010) XDS. Acta Crystallogr., Sect. D: Biol. Crystallogr. 66, 125-132.

(36) Evans, P. R., and Murshudov, G. N. (2013) How good are my data and what is the resolution? Acta Crystallogr., Sect. D: Biol. Crystallogr. 69, 1204-1214.

(37) McCoy, A. J., Grosse-Kunstleve, R. W., Adams, P. D., Winn, M. D., Storoni, L. C., and Read, R. J. (2007) Phaser crystallographic software. J. Appl. Crystallogr. 40, 658-674.

(38) Adams, P. D., Afonine, P. V., Bunkoczi, G., Chen, V. B., Davis, I. W., Echols, N., Headd, J. J., Hung, L. W., Kapral, G. J., GrosseKunstleve, R. W., McCoy, A. J., Moriarty, N. W., Oeffner, R., Read, R. J., Richardson, D. C., Richardson, J. S., Terwilliger, T. C., and Zwart, P. H. (2010) PHENIX: a comprehensive Python-based system for macromolecular structure solution. Acta Crystallogr., Sect. D: Biol. Crystallogr. 66, 213-221.

(39) Emsley, P., Lohkamp, B., Scott, W. G., and Cowtan, K. (2010) Features and development of Coot. Acta Crystallogr., Sect. D: Biol. Crystallogr. 66, 486-501. 